\title{
A Mixed Integer Linear Program for Airport Departure Scheduling
}

\author{
Gautam Gupta \\ University of Santa Cruz \\ NASA Ames Research Center, Moffett Field, CA 94035 \\ Yoon C Jung \\ NASA Ames Research Center, Moffett Field, CA 94035
}

\begin{abstract}
Aircraft departing from an airport are subject to numerous constraints while scheduling departure times. These constraints include wake-separation constraints for successive departures, miles-in-trail separation for aircraft bound for the same departure fixes, and time-window or prioritization constraints for individual flights. Besides these, emissions as well as increased fuel consumption due to inefficient scheduling need to be included. Addressing all the above constraints in a single framework while allowing for resequencing of the aircraft using runway queues is critical to the implementation of the Next Generation Air Transport System (NextGen) concepts.
\end{abstract}

Prior work on airport departure scheduling has addressed some of the above. However, existing methods use pre-determined runway queues, and schedule aircraft from these departure queues. The source of such pre-determined queues is not explicit, and could potentially be a subjective controller input. Determining runway queues and scheduling within the same framework would potentially result in better scheduling. This paper presents a mixed integer linear program (MILP) for the departure-scheduling problem. The program takes as input the incoming sequence of aircraft for departure from a runway, along with their earliest departure times and an optional prioritization scheme based on time-window of departure for each aircraft. The program then assigns these aircraft to the available departure queues and schedules departure times, explicitly considering wake separation and departure fix restrictions to minimize total delay for all aircraft. The approach is generalized and can be used in a variety of situations, and allows for aircraft prioritization based on operational as well as environmental considerations. We present the MILP in the paper, along with benefits over the first-come-first-serve (FCFS) scheme for numerous randomized problems based on real-world settings. The MILP results in substantially reduced delays as compared to FCFS, and the magnitude of the savings depends on the queue and departure fix structure.

The MILP assumes deterministic aircraft arrival times at the runway queues. However, due to taxi time uncertainty, aircraft might arrive either earlier or later than these deterministic times. Thus, to incorporate this uncertainty, we present a method for using the MILP with "overlap discounted rolling planning horizon". The approach is based on valuing near-term decision results more than future ones. We develop a model of taxitime uncertainty based on real-world data, and then compare the baseline FCFS delays with delays using the above MILP in a simple rolling-horizon method and in the overlap discounted scheme. 